\title{
Prognostic Impact of Computed Tomography-Derived Abdominal Fat Area on Transcatheter Aortic Valve Implantation
}

\author{
Taishi Okuno, MD; Keita Koseki, MD; Toru Nakanishi, MD; Kai Ninomiya, MD; \\ Daijiro Tomii, MD; Tetsu Tanaka, MD; Yu Sato, MD; Akira Osanai, MD; Kei Sato, MD; \\ Hideki Koike, MD; Kazuyuki Yahagi, MD; Satoru Kishi, MD; Kota Komiyama, MD; \\ Jiro Aoki, MD, PhD; Motoi Yokozuka, MD; Sumio Miura, MD; Kengo Tanabe, MD, PhD
}

\begin{abstract}
Background: Obesity has previously been identified as an indicator of good prognosis in patients undergoing transcatheter aortic valve implantation (TAVI), an association known as the "obesity paradox". We investigated whether abdominal total fat area (TFA), visceral fat area (VFA), or subcutaneous fat area (SFA) are prognostic indicators of long-term clinical outcome in patients undergoing TAVI.

Methods and Results: We retrospectively analyzed 100 consecutive patients who underwent TAVI between December 2013 and April 2017. TFA, VFA, and SFA were measured from routine pre-procedural computed tomography (CT). Patients were divided into 2 groups according to median TFA, VFA, or SFA, and we investigated the association of abdominal fat area with adverse clinical events, including all-cause death and re-hospitalization due to worsening heart failure. At a median follow-up of 665 days, patients with higher SFA had significantly lower incidence of the composite outcome and all-cause death compared with patients with lower SFA ( $15.0 \%$ vs. $37.7 \%, P=0.025$; and $8.9 \%$ vs. $23.7 \%, P=0.047$, respectively). In contrast, patients with higher TFA or VFA did not show significant reduction in the incidences of the composite outcome or all-cause mortality.
\end{abstract}

Conclusions: CT-derived SFA had prognostic value in patients undergoing TAVI.

Key Words: Computed tomography; Obesity paradox; Subcutaneous fat area; Transcatheter aortic valve implantation

$\mathbf{T}$ ranscatheter aortic valve implantation (TAVI) is a widely accepted therapeutic option for symptomatic severe aortic valve stenosis (AS), especially in highrisk patients such as elderly or frail patients, and those with multiple comorbidities. Given that patients in this group have a higher intrinsic risk, TAVI has been reported to be associated with a relatively high rate of long-term mortality and re-hospitalization due to heart failure. ${ }^{1}$ Therefore, it is important to identify patients at higher risk who may not benefit from this procedure.

Recently, body mass index (BMI) has been identified as an important prognostic indicator in patients undergoing TAVI. The "obesity paradox" refers to the paradoxical finding that higher BMI is an indicator of good prognosis not only in patients undergoing TAVI but also in patients with various diseases including diabetes, renal disease, stroke, and other cardiovascular diseases. ${ }^{2-15}$ BMI, however, is of potentially limited clinical value due to its inability to differentiate between fat mass and lean body mass, and there have been no reports on the individual prognostic value of body fat.

The aim of the present study was therefore to evaluate the effect of abdominal fat on long-term clinical outcomes in patients undergoing TAVI. To clarify this, we focused on abdominal fat area, including the visceral fat area (VFA) and subcutaneous fat area (SFA) evaluated on computed tomography (CT), and evaluated their individual effects on long-term clinical outcomes such as all-cause mortality and re-hospitalization due to worsening heart failure in patients undergoing TAVI.

\section{Methods}

\section{Subjects}

This was a retrospective study of 100 consecutive patients with severe AS undergoing TAVI between December 2013 and April 2017 at Mitsui Memorial Hospital in Tokyo, Japan.

Received June 21, 2018; revised manuscript received August 13, 2018; accepted September 4, 2018; released online October 5, 2018 Time for primary review: 40 days

Division of Cardiology (T.O., K. Koseki, T.N., K.N., D.T., T.T., Y.S., K.S., H.K., K.Y., K. Komiyama, J.A., K.T.), Division of Cardiovascular Surgery (A.O., S.M.), Division of Anesthesia (M.Y.), Mitsui Memorial Hospital, Tokyo; Division of General Internal Medicine, Nakahara Medical Clinic, Kawasaki (S.K.), Japan

Mailing address: Kengo Tanabe, MD, PhD, Director of Division of Cardiology, Mitsui Memorial Hospital, 1 Kanda-Izumicho, Chiyoda-ku, Tokyo 101-8643, Japan. E-mail: kengo-t@zd5.so-net.ne.jp

ISSN-1346-9843 All rights are reserved to the Japanese Circulation Society. For permissions, please e-mail: cj@j-circ.or.jp 


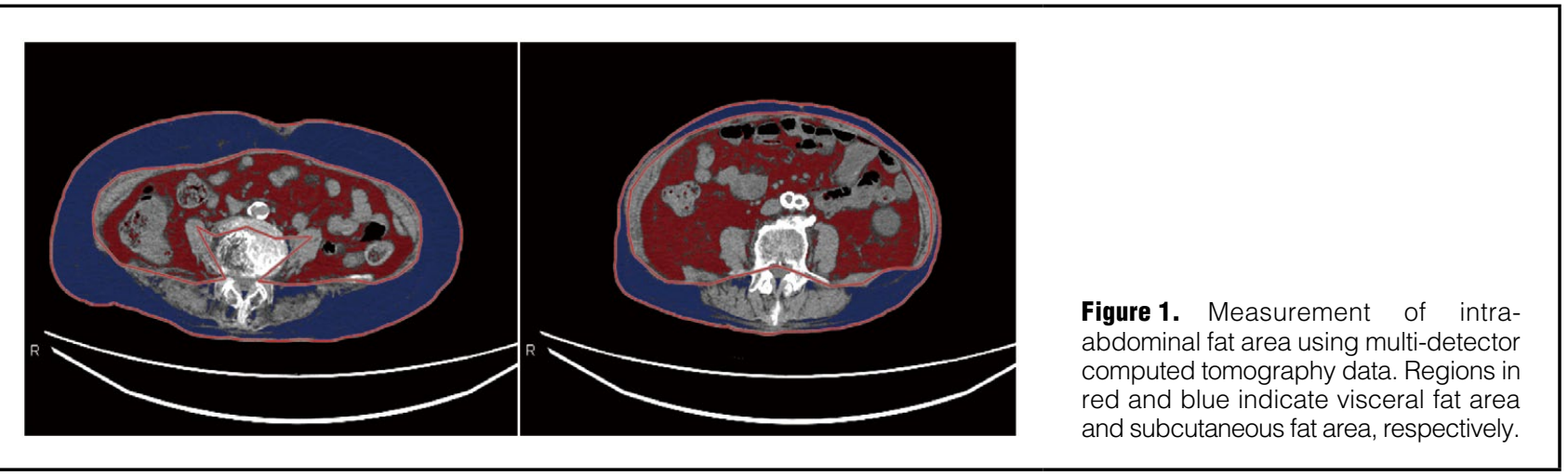

The multidisciplinary heart team discussed all cases, and a consensus was achieved regarding the therapeutic strategy in each case. TAVI was performed in a hybrid operating room under general anesthesia using balloon-expandable or self-expandable transcatheter aortic valves. All patients provided written informed consent prior to study enrollment and the identities of these patients have been protected.

\section{Measurement of VFA and SFA}

All patients underwent routine pre-procedural $\mathrm{CT}$, which was performed with a 320-row multi-detector CT (Aquilion ONE, Canon Medical Systems, Tochigi, Japan) with patients placed in the decubitus dorsalis position. The images obtained were transferred to a dedicated workstation (Ziostation2, Ziosoft, Tokyo, Japan), in which the total fat area (TFA), VFA, and SFA were semi-automatically measured at the level of the umbilicus (Figure 1).

\section{Endpoints and Follow-up}

The occurrence of clinical events was recorded from admission and outpatient medical records or by telephone interview. All patient follow-up data were collected by March 2018. The median follow-up period was 665 days (IQR, 390-956 days). Endpoints were composite outcomes of all-cause mortality and re-hospitalization due to worsening heart failure. For patients who had multiple cardiac events during the study period, the time until the first event was used in the calculations.

\section{Statistical Analysis}

Categorical data are represented as frequencies and percentages and the differences between groups were evaluated with the chi-squared test. Continuous variables were tested for normal distribution using the Kolmogorov-Smirnov test. Non-normally distributed variables are expressed as median (IQR) and were compared between groups using the Mann-Whitney U-test. Normally distributed variables are expressed as mean \pm SD and were compared between groups using Student's t-test. Spearman's rank correlation coefficients (r) were calculated to assess the link and the degree of association between BMI and abdominal fat area. Event-free survival curves were constructed using the Kaplan-Meier method and compared using the log-rank test. Univariate and multivariate Cox proportional hazards models were used to calculate hazard ratios (HR) and 95\% CI for the composite outcome and all-cause mortality. Because this was an observational study and differences in baseline characteristics could significantly affect outcome, sensitivity analysis was performed to adjust for confounders using 2 Cox regression models. The first model was adjusted for age and gender, both of which should be considered for abdominal fat area. In the second model, we selected covariates that were different $(\mathrm{P}<0.1)$ between patients with and without the composite outcome. In this model, we used a stepwise-backward selection with a probability to remove the effect from the regression at $\mathrm{P}>0.05$. Throughout the present study, $\mathrm{P}<0.05$ was considered significant. Statistical analysis was performed using EZR (Saitama Medical Center, Jichi Medical University, Saitama, Japan), which is a graphical user interface for R (R Foundation for Statistical Computing, Vienna, Austria).

\section{Results}

Baseline Characteristics According to Composite Outcome Baseline patient characteristics are listed in Table 1. During the follow-up period, 34 patients experienced clinical events, including 20 cases of all-cause mortality and 20 cases of re-hospitalization due to worsening heart failure.

The composite outcome was observed in patients who had a significantly higher prevalence of atrial fibrillation, significantly lower hemoglobin, and significantly higher brain natriuretic peptide. There were no differences in body weight, BMI, waist circumference (WC), or body surface area (BSA) between patients who reached the composite outcome and those who did not. In terms of CT-derived abdominal fat area, SFA was numerically lower in patients who reached the composite outcome, whereas VFA was similar between patients who reached the composite outcome and those who did not.

\section{Baseline Characteristics According to SFA}

We divided all patients into 2 groups according to median SFA: the low-SFA group (SFA $<92.6 \mathrm{~cm}^{2}, \mathrm{n}=50$ ) and the high-SFA group (SFA $\geq 92.6 \mathrm{~cm}^{2}, \mathrm{n}=50$ ). Baseline characteristics are listed in Table 2. As expected, the low-SFA group had lower body weight, WC, BMI, BSA, TFA, and VFA. There were no significant differences in other baseline characteristics, including age, gender, clinical status, concomitant diseases, previous medical history, laboratory data, echocardiographic data, and procedures.

\section{BMI and Abdominal Fat Area}

We assessed the correlation between BMI and abdominal fat area (Figure 2). BMI had a positive and statistically 
Table 1. Baseline Patient Characteristics vs. Composite Outcome

\begin{tabular}{|c|c|c|c|c|}
\hline & $\begin{array}{l}\text { All patients } \\
(n=100)\end{array}$ & $\begin{array}{l}\text { Composite outcome }(+) \\
\qquad(n=34)\end{array}$ & $\begin{array}{l}\text { Composite outcome (-) } \\
\qquad(n=66)\end{array}$ & P-value \\
\hline Age (years) & $84(81-88)$ & $84(80-89)$ & $85(81-87)$ & 0.813 \\
\hline Female & $69(69.0)$ & 25 (73.5) & $44(66.7)$ & 0.649 \\
\hline Height (cm) & $151.2 \pm 8.2$ & $150.4 \pm 8.8$ & $151.6 \pm 7.9$ & 0.515 \\
\hline Weight (kg) & $50.4 \pm 9.7$ & $49.7 \pm 8.7$ & $50.7 \pm 10.2$ & 0.618 \\
\hline WC (cm) & $79.5(71.8-87.3)$ & $78.7(72.5-84.1)$ & $80.5(71.4-87.6)$ & 0.485 \\
\hline BMI $\left(\mathbf{k g} / \mathbf{m}^{2}\right)$ & $22.0 \pm 4.0$ & $21.8 \pm 4.6$ & $22.0 \pm 3.8$ & 0.800 \\
\hline BSA $\left(m^{2}\right)$ & $1.45 \pm 0.15$ & $1.44 \pm 0.14$ & $1.46 \pm 0.16$ & 0.527 \\
\hline \multicolumn{5}{|l|}{ CT-derived abdominal fat area $\left(\mathrm{cm}^{2}\right)$} \\
\hline TFA & 174.4 (56.4-255.2) & $131.6(52.2-237.3)$ & $183.8(62.6-264.2)$ & 0.291 \\
\hline SFA & $92.6(32.9-144.0)$ & $61.7(35.0-126.45)$ & $100.1(32.2-148.8)$ & 0.188 \\
\hline VFA & $63.0(23.3-107.9)$ & $64.7(14.0-108.8)$ & $63.0(29.5-106.9)$ & 0.678 \\
\hline Logistic EuroSCORE (\%) & $10.43(7.01-17.47)$ & $13.57(8.48-21.27)$ & $10.11(6.96-15.21)$ & 0.075 \\
\hline STS score: mortality (\%) & $5.26(3.96-7.10)$ & $5.00(3.76-7.54)$ & $5.44(3.97-6.91)$ & 0.949 \\
\hline NYHA functional class III/IV & $38(38.0)$ & $14(41.2)$ & $24(36.4)$ & 0.668 \\
\hline \multicolumn{5}{|l|}{ Concomitant disease } \\
\hline Hypertension & $69(69.0)$ & $25(73.5)$ & $44(66.7)$ & 0.649 \\
\hline Diabetes & $25(25.0)$ & $6(17.6)$ & $19(28.8)$ & 0.330 \\
\hline Dyslipidemia & $57(57.0)$ & $17(50.0)$ & $40(60.6)$ & 0.394 \\
\hline CKD (eGFR <60 mL/min/1.73 m²) & $35(35.0)$ & $13(38.2)$ & $22(33.3)$ & 0.662 \\
\hline COPD & $10(10.0)$ & $5(14.7)$ & $5(7.6)$ & 0.301 \\
\hline Atrial fibrillation & $27(27.0)$ & $14(41.2)$ & $13(19.7)$ & 0.032 \\
\hline \multicolumn{5}{|l|}{ Previous history } \\
\hline Previous MI & $2(2.0)$ & $0(0.0)$ & $2(3.0)$ & 0.547 \\
\hline Prior stroke & $14(14.0)$ & $8(23.5)$ & $6(9.1)$ & 0.068 \\
\hline Previous PAD & $2(2.0)$ & $0(0.0)$ & $2(3.0)$ & 0.547 \\
\hline \multicolumn{5}{|l|}{ Laboratory data (before procedure) } \\
\hline Hemoglobin $(\mathrm{g} / \mathrm{dL})$ & $10.9(9.7-12.3)$ & $10.0(9.2-11.1)$ & $11.4(10.2-12.5)$ & 0.006 \\
\hline WBC $(/ \mu \mathrm{L})$ & $4,900(4,100-6,600)$ & $4,500(3,900-6,470)$ & $5,100(4,300-6,600)$ & 0.118 \\
\hline Platelet count $\left(10^{4} / \mu \mathrm{L}\right)$ & $18.7(14.5-21.4)$ & $17.1(13.8-21.3)$ & $18.9(14.7-21.4)$ & 0.291 \\
\hline eGFR (mL/min/1.73 m²) & $54.6(38.6-64.4)$ & $48.9(38.9-64.6)$ & $56.2(37.1-64.2)$ & 0.603 \\
\hline ALB $(g / d L)$ & $4.1(3.7-4.3)$ & $3.9(3.6-4.3)$ & $4.2(3.7-4.4)$ & 0.065 \\
\hline $\mathrm{T}-\mathrm{CHO}(\mathrm{mg} / \mathrm{dL})$ & $176.0(151.5-195.0)$ & $164.5(147.5-188.8)$ & $177.5(153.5-195.8)$ & 0.522 \\
\hline $\mathrm{Na}(\mathrm{mEq} / \mathrm{L})$ & $139(137-142)$ & $139(136-141)$ & $140(137-142)$ & 0.206 \\
\hline $\mathrm{K}(\mathrm{mEq} / \mathrm{L})$ & $4.4(4.0-4.8)$ & $4.4(4.1-4.8)$ & $4.4(3.9-4.8)$ & 0.461 \\
\hline BNP (pg/mL) & $183.7(115.3-446.3)$ & $333.5(151.1-561.1)$ & $164.9(112.2-363.8)$ & 0.049 \\
\hline \multicolumn{5}{|l|}{ Echocardiographic data } \\
\hline \multicolumn{5}{|l|}{ Before procedure } \\
\hline LVEF (\%) & $65.2 \pm 14.5$ & $63.6 \pm 14.0$ & $66.1 \pm 14.8$ & 0.425 \\
\hline LVEF $\leq 35 \%$ & $6(6.0)$ & $1(2.9)$ & $5(7.7)$ & 0.661 \\
\hline Calculated AVA (cm²) & $0.65 \pm 0.18$ & $0.67 \pm 0.19$ & $0.65 \pm 0.18$ & 0.617 \\
\hline Mean aortic gradient $(\mathrm{mmHg})$ & $58.6 \pm 18.4$ & $54.6 \pm 19.5$ & $60.7 \pm 17.6$ & 0.117 \\
\hline Peak jet velocity $(\mathrm{m} / \mathrm{s})$ & $4.78 \pm 0.77$ & $4.58 \pm 0.83$ & $4.88 \pm 0.72$ & 0.061 \\
\hline Aortic regurgitation $\geq$ moderate & $12(12.0)$ & $5(14.7)$ & $7(10.8)$ & 0.747 \\
\hline \multicolumn{5}{|l|}{ Procedural data } \\
\hline Trans-femoral approach & $87(87.0)$ & $28(82.4)$ & $59(89.4)$ & 0.356 \\
\hline Balloon-expandable valves (XT/S3) & $86(86.0)$ & $31(91.2)$ & $55(83.3)$ & 0.371 \\
\hline \multicolumn{5}{|l|}{ After procedure } \\
\hline Calculated AVA $\left(\mathrm{cm}^{2}\right)$ & $1.63 \pm 0.43$ & $1.52 \pm 0.39$ & $1.68 \pm 0.45$ & 0.099 \\
\hline Mean aortic gradient $(\mathrm{mmHg})$ & $11.8 \pm 5.2$ & $11.2 \pm 5.1$ & $12.0 \pm 5.3$ & 0.481 \\
\hline Peak jet velocity $(\mathrm{m} / \mathrm{s})$ & $2.17 \pm 0.42$ & $2.09 \pm 0.42$ & $2.20 \pm 0.42$ & 0.206 \\
\hline Aortic regurgitation $\geq$ moderate & $4(4.0)$ & $1(2.9)$ & $3(4.6)$ & 1.000 \\
\hline
\end{tabular}

Data given as mean $\pm S D$, median (IQR) or $n(\%)$. ALB, albumin; AVA, aortic valve area; BNP, brain natriuretic peptide; BMI, body mass index; $\mathrm{BSA}$, body surface area; CABG, coronary artery bypass graft; CKD, chronic kidney disease; COPD, chronic obstructive pulmonary disease; CT, computed tomography; eGFR, estimated glomerular filtration rate; EuroSCORE, European System for Cardiac Operative Risk Evaluation; K, potassium; LVEF, left ventricular ejection fraction; MI, myocardial infarction; Na, sodium; NYHA, New York Heart Association; PAD, peripheral artery disease; PCl, percutaneous coronary intervention; S3, SAPIEN 3 (Edwards Lifesciences, Irvine, CA, USA); SFA, subcutaneous fat area; STS, Society of Thoracic Surgeons; T-CHO, total cholesterol; TFA, total fat area; VFA, visceral fat area; WBC, white blood cell count; WC, waist circumference; XT, SAPIEN XT (Edwards Lifesciences, Irvine, CA, USA). 


\begin{tabular}{|c|c|c|c|}
\hline & $\begin{array}{c}\text { Low-SFA group } \\
\qquad(n=50)\end{array}$ & $\begin{array}{l}\text { High-SFA group } \\
\qquad(n=50)\end{array}$ & P-value \\
\hline Age (years) & $85(81-89)$ & $84(80-87)$ & 0.189 \\
\hline Female & $30(60)$ & $39(78)$ & 0.083 \\
\hline Height (cm) & $151.8 \pm 9.2$ & $150.6 \pm 7.1$ & 0.445 \\
\hline Weight (kg) & $44.7 \pm 8.1$ & $56.1 \pm 7.6$ & $<0.001$ \\
\hline WC (cm) & $71.6(67.1-76.2)$ & $86.9(81.7-91.1)$ & $<0.001$ \\
\hline BMI $\left(\mathbf{k g} / \mathbf{m}^{2}\right)$ & $19.3 \pm 2.5$ & $24.6 \pm 3.5$ & $<0.001$ \\
\hline BSA $\left(m^{2}\right)$ & $1.39 \pm 0.16$ & $1.52 \pm 0.12$ & $<0.001$ \\
\hline \multicolumn{4}{|l|}{ CT-derived abdominal fat area $\left(\mathrm{cm}^{2}\right)$} \\
\hline TFA & $55.7(19.4-115.3)$ & $250.9(198.0-322.0)$ & $<0.001$ \\
\hline SFA & $31.4(10.1-59.3)$ & $144.9(117.7-193.9)$ & $<0.001$ \\
\hline VFA & $23.0(8.9-53.9)$ & $96.5(63.6-135.4)$ & $<0.001$ \\
\hline Logistic EuroSCORE (\%) & $10.11(6.59-17.60)$ & $10.74(7.48-16.62)$ & 0.893 \\
\hline STS score: mortality (\%) & $5.86(3.91-7.29)$ & $4.81(3.97-7.01)$ & 0.279 \\
\hline NYHA functional class III/IV & $18(36)$ & $20(40)$ & 0.837 \\
\hline \multicolumn{4}{|l|}{ Concomitant disease } \\
\hline Hypertension & $36(72)$ & $33(66)$ & 0.666 \\
\hline Diabetes & $11(22)$ & $14(28)$ & 0.645 \\
\hline Dyslipidemia & $25(25)$ & $32(64)$ & 0.225 \\
\hline CKD (eGFR <60 mL/min/1.73 m²) & $16(32)$ & $19(38)$ & 0.675 \\
\hline COPD & $4(8)$ & $6(12)$ & 0.741 \\
\hline Atrial fibrillation & $15(30)$ & $12(24)$ & 0.653 \\
\hline \multicolumn{4}{|l|}{ Previous history } \\
\hline Previous MI & $1(2)$ & $1(2)$ & 1.000 \\
\hline Prior stroke & $9(18)$ & $5(10)$ & 0.388 \\
\hline Previous PAD & $1(2)$ & $1(2)$ & 1.000 \\
\hline Laboratory data (before procedure) & & & 1.000 \\
\hline Hemoglobin (g/dL) & $10.8(9.4-12.5)$ & $11.1(10.0-12.2)$ & 0.809 \\
\hline WBC $(/ \mu \mathrm{L})$ & $4,700(4,030-6,570)$ & $4,950(4,120-6,750)$ & 0.748 \\
\hline Platelet count $\left(10^{4} / \mu \mathrm{L}\right)$ & $18.7(14.5-21.4)$ & $18.5(14.3-21.9)$ & 0.937 \\
\hline eGFR (mL/min/1.73 m²) & $55.5(39.8-65.7)$ & $53.2(35.8-62.1)$ & 0.252 \\
\hline ALB $(g / d L)$ & $4.0(3.7-4.4)$ & $4.1(3.7-4.3)$ & 0.616 \\
\hline $\mathrm{T}-\mathrm{CHO}(\mathrm{mg} / \mathrm{dL})$ & $179.5(153.5-196.0)$ & $176.0(150.5-185.8)$ & 0.756 \\
\hline $\mathrm{Na}(\mathrm{mEq} / \mathrm{L})$ & $139(135-141)$ & $140(137-142)$ & 0.315 \\
\hline $\mathrm{K}(\mathrm{mEq} / \mathrm{L})$ & $4.5(4.2-4.9)$ & $4.4(4.0-4.6)$ & 0.241 \\
\hline BNP (pg/mL) & $210.6(127.5-436.0)$ & $163.7(104.5-441.3)$ & 0.632 \\
\hline \multicolumn{4}{|l|}{ Echocardiographic data } \\
\hline \multicolumn{4}{|l|}{ Before procedure } \\
\hline LVEF (\%) & $65.2 \pm 14.6$ & $65.2 \pm 14.5$ & 0.977 \\
\hline LVEF $\leq 35 \%$ & $2(4)$ & $4(8)$ & 0.436 \\
\hline Calculated AVA $\left(\mathrm{cm}^{2}\right)$ & $0.65 \pm 0.17$ & $0.66 \pm 0.19$ & 0.817 \\
\hline Mean aortic gradient $(\mathrm{mmHg})$ & $57.8 \pm 19.0$ & $59.4 \pm 18.0$ & 0.662 \\
\hline Peak jet velocity $(\mathrm{m} / \mathrm{s})$ & $4.74 \pm 0.79$ & $4.18 \pm 0.75$ & 0.628 \\
\hline Aortic regurgitation $\geq$ moderate & $7(14)$ & $5(10)$ & 0.760 \\
\hline \multicolumn{4}{|l|}{ Procedural data } \\
\hline Trans-femoral approach & $41(82)$ & $46(92)$ & 0.234 \\
\hline Balloon-expandable valves (XT/S3) & $44(88)$ & $42(84)$ & 0.774 \\
\hline \multicolumn{4}{|l|}{ After procedure } \\
\hline Calculated AVA $\left(\mathrm{cm}^{2}\right)$ & $1.56 \pm 0.44$ & $1.69 \pm 0.43$ & 0.174 \\
\hline Mean aortic gradient $(\mathrm{mmHg})$ & $11.4 \pm 5.0$ & $12.1 \pm 5.4$ & 0.557 \\
\hline Peak jet velocity $(\mathrm{m} / \mathrm{s})$ & $2.16 \pm 0.43$ & $2.17 \pm 0.41$ & 0.927 \\
\hline Aortic regurgitation $\geq$ moderate & $4(8)$ & $0(0)$ & 0.117 \\
\hline
\end{tabular}

Data given as mean \pm SD, median (IQR) or $n(\%)$. Abbreviations as in Table 1. 

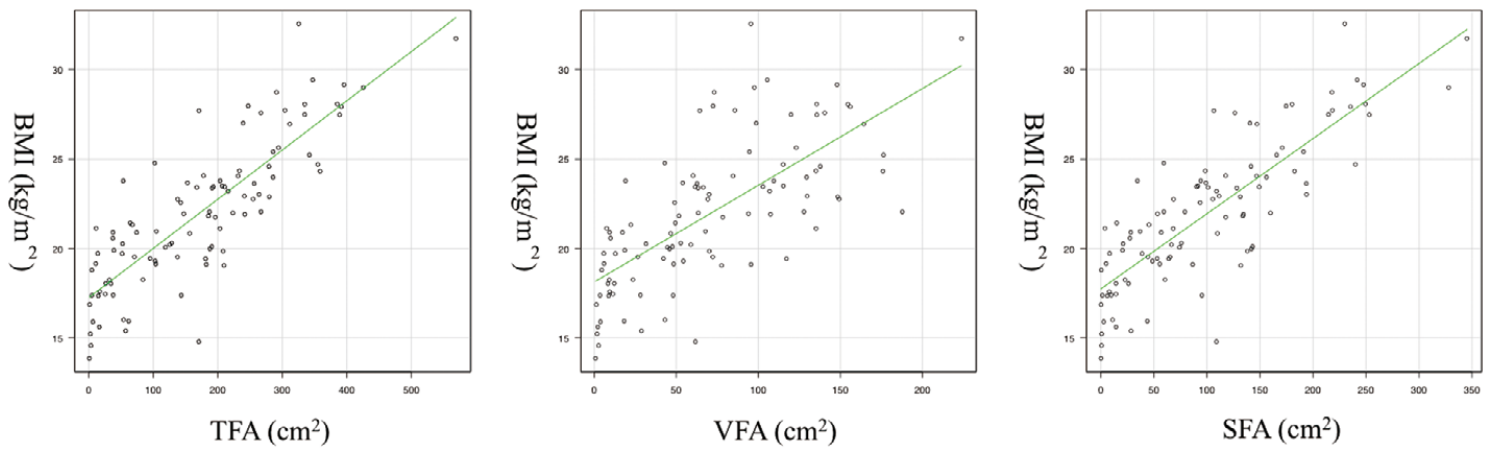

Figure 2. Body mass index (BMI) vs. total fat area (TFA), visceral fat area (VFA), and subcutaneous fat area (SFA).
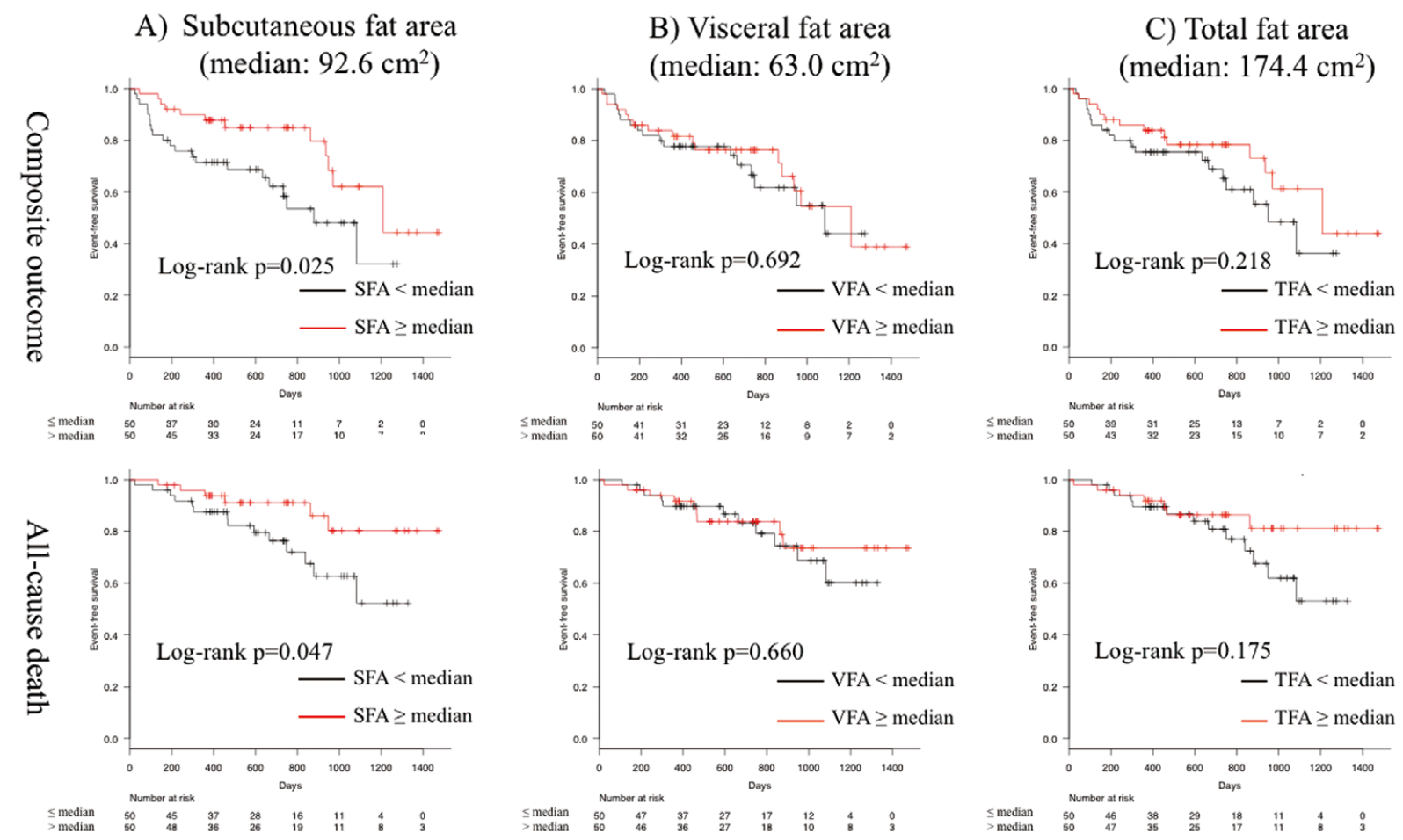

Figure 3. Kaplan-Meier analysis for the composite outcome, including all-cause mortality and re-hospitalization due to worsening heart failure and all-cause mortality according to median (A) subcutaneous fat area (SFA), (B) visceral fat area (VFA), and (C) total fat area (TFA).

significant correlation with TFA $(\mathrm{r}=0.832, \mathrm{P}<0.001)$, VFA $(\mathrm{r}=0.747, \mathrm{P}<0.001)$, and SFA $(\mathrm{r}=0.807, \mathrm{P}<0.001)$.

\section{Abdominal Fat Area and Long-Term Clinical Outcome}

Cumulative incidence of the composite outcome and allcause mortality were compared between patients with lower and higher SFA, VFA, or TFA. The Kaplan-Meier survival curves for the composite outcome and all-cause mortality are shown in Figure 3. During a median follow-up period of 665 days, lower SFA was significantly associated with higher cumulative incidence of the composite outcome
(37.7\% vs. $15.0 \%, \mathrm{P}=0.025)$ and all-cause mortality $(23.7 \%$ vs. $8.9 \%, \mathrm{P}=0.047)$. In contrast, lower VFA had no significant effect on the cumulative incidence of composite outcome $(29.5 \%$ vs. $23.6 \%, \mathrm{P}=0.692)$ or all-cause mortality (16.7\% vs. $16.2 \%, \mathrm{P}=0.66)$. Consequently, lower TFA had a tendency to be associated with a higher cumulative incidence of the composite outcome (31.2\% vs. $21.7 \%$, $\mathrm{P}=0.218)$ and all-cause mortality $(19.2 \%$ vs. $13.7 \%, \mathrm{P}=0.175)$. Statistical significance, however, was not observed. Similarly, lower BMI, lower BSA, and lower WC were not significantly associated with the composite outcome. For 


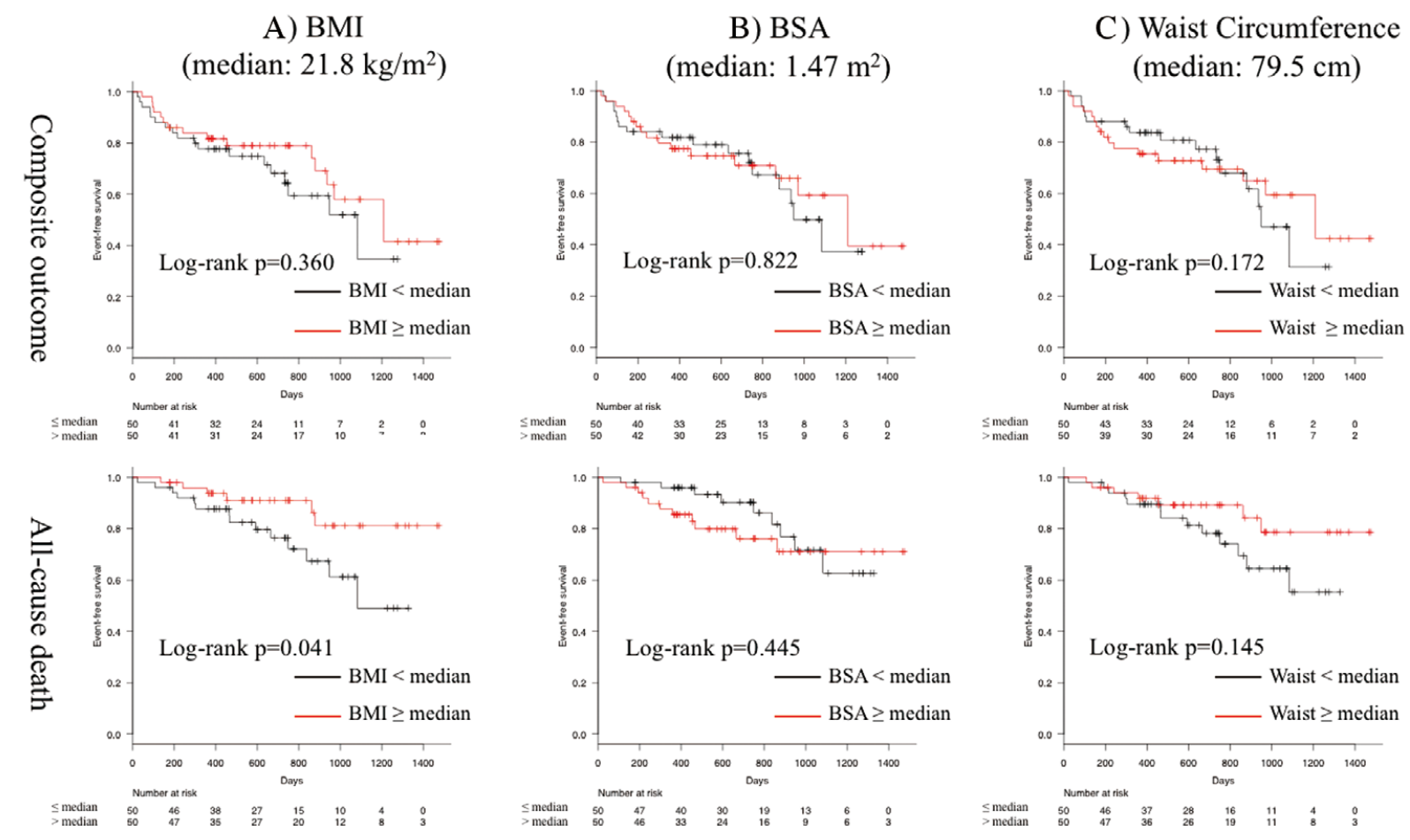

Figure 4. Kaplan-Meier analysis for the composite outcome, including all-cause mortality and re-hospitalization due to worsening heart failure and all-cause mortality according to median (A) body mass index (BMI), (B) body surface area (BSA), and (C) waist circumference.

\begin{tabular}{|c|c|c|c|c|c|c|}
\hline & \multicolumn{2}{|c|}{ Univariate } & \multicolumn{2}{|c|}{ Model 1} & \multicolumn{2}{|c|}{ Model 2} \\
\hline & HR $(95 \% \mathrm{Cl})$ & P-value & HR (95\% Cl) & P-value & HR $(95 \% \mathrm{Cl})$ & P-value \\
\hline Lower SFA & $2.18(1.08-4.40)$ & 0.029 & $2.12(1.04-4.34)$ & 0.039 & $2.77(1.27-6.02)$ & 0.010 \\
\hline Age & $1.03(0.97-1.09)$ & 0.390 & $1.01(0.95-1.08)$ & 0.727 & \multicolumn{2}{|c|}{ Not included } \\
\hline Female & $0.82(0.38-1.76)$ & 0.611 & $0.83(0.38-1.80)$ & 0.639 & \multicolumn{2}{|c|}{ Not included } \\
\hline Logistic EuroSCORE & $1.02(0.98-1.06)$ & 0.418 & & & \multicolumn{2}{|c|}{ Dropped } \\
\hline Atrial fibrillation & $2.06(1.03-4.10)$ & 0.040 & & & \multicolumn{2}{|c|}{ Dropped } \\
\hline Prior stroke & $2.34(1.05-5.24)$ & 0.039 & & & $2.65(1.13-6.18)$ & 0.025 \\
\hline Hemoglobin (g/dL) & $0.74(0.59-0.93)$ & 0.010 & & & $0.73(0.57-0.92)$ & 0.009 \\
\hline ALB $(\mathrm{g} / \mathrm{dL})$ & $0.49(0.24-0.98)$ & 0.045 & & & \multicolumn{2}{|l|}{ Dropped } \\
\hline BNP (pg/mL) & $1.00(1.00-1.00)$ & 0.010 & & & \multicolumn{2}{|c|}{ Dropped } \\
\hline Post-procedure AVA & $0.53(0.23-1.20)$ & 0.128 & & & \multicolumn{2}{|c|}{ Dropped } \\
\hline
\end{tabular}

Model 1, adjusted for age and gender. Model 2, adjusted for Logistic EuroSCORE, atrial fibrillation, prior stroke, hemoglobin, ALB, BNP, calculated post-procedure AVA, using stepwise-backward selection. Abbreviations as in Table 1.

all-cause mortality, only lower BMI was associated with higher cumulative incidence $(23.6 \%$ vs. $9.0 \%, \mathrm{P}=0.041$; Figure 4).

The results of the Cox regression analysis for the cumulative incidence of the composite outcome are given in Table 3. On Cox univariate analysis, the HR for the composite outcome or all-cause mortality were $2.18(95 \%$ CI: $1.08-4.40, \mathrm{P}=0.029)$ and 2.56 (95\% CI: 0.98-6.67, $\mathrm{P}=0.055)$ for the lower SFA group, respectively. After adjusting for age and gender, lower SFA was independently associated with higher risk of the composite outcome (HR,
2.12; 95\% CI: 1.04 4.34, $\mathrm{P}=0.039)$. Likewise, on stepwise Cox regression modeling, there was an increased risk of the composite outcome in the lower SFA group (HR, 2.77; 95\% CI: $1.27-6.02, \mathrm{P}=0.010)$.

\section{Discussion}

The present study has demonstrated that SFA was significantly associated with long-term clinical outcome in patients undergoing TAVI, whereas VFA and TFA were not. To the best of our knowledge, this is the first study to evaluate 
the relationship between abdominal fat area and long-term clinical outcomes in patients undergoing TAVI.

Pre-procedural CT is currently considered the gold standard for the evaluation of patients undergoing TAVI, and is now widely used as a routine pre-procedural evaluation at a number of institutions. ${ }^{16-19}$ Therefore, SFA, which can be easily measured on pre-procedural CT, ${ }^{20-22}$ could be a simple and useful prognostic indicator in patients undergoing TAVI.

Previous studies using BMI as a measure of obesity have demonstrated an obesity paradox in patients undergoing TAVI, as well as in patients undergoing percutaneous coronary intervention (PCI) or surgical aortic valve replacement (SAVR). ${ }^{9-11} \mathrm{BMI}$, however, is a non-specific parameter that is potentially limited by the inability to differentiate between fat mass and lean body mass. Moreover, although BMI has a positive correlation with abdominal fat area, BMI cannot differentiate visceral fat mass from subcutaneous fat mass. Therefore, it has been unclear what component of BMI affects clinical outcome in patients undergoing TAVI. The present study suggests that not visceral fat but subcutaneous fat has prognostic value in patients undergoing TAVI.

Possible explanations for the obesity paradox have been discussed in previous studies. Given that obesity measured by BMI includes both lean body mass and fat mass, higher lean body mass, which consists of skeletal muscle mass, was suggested to be one of the reasons for the obesity paradox. Namely, lower skeletal muscle mass is correlated with frailty and was suggested to be associated with early poor outcome and high-resource utilization after TAVI. ${ }^{23}$ The present study, however, suggests that fat mass, especially subcutaneous fat mass, might also have prognostic value. This provides another possible mechanism for the obesity paradox in patients undergoing TAVI. First, the aging population of patients who tend to undergo TAVI might be different from other populations in which the obesity paradox has been previously suggested. In the present study, the median VFA and SFA for all patients were $63.0 \mathrm{~cm}^{2}$ (IQR, 23.2-107.9 $\mathrm{cm}^{2}$ ) and $92.6 \mathrm{~cm}^{2}$ (IQR, 32.9$\left.144.0 \mathrm{~cm}^{2}\right)$, respectively, which are far lower than the average reported in previous studies. ${ }^{\mathbf{2 4 , 2 5}}$ This suggests that the aging population of patients undergoing TAVI may have lower baseline body fat. Therefore, lower SFA in this population might suggest excessive body fat loss, which indicates malnutrition, multi-organ dysfunction, or multiple comorbidities including occult malignancy. These factors have been identified as significant predictors of mortality in other populations, ${ }^{\mathbf{2 6 - 2 9}}$ and could therefore be applicable to the present situation. In this context, this mechanism seems to be applicable not only to TAVI patients but also to general elderly patients. To our knowledge, however, there are no studies limited to elderly patients that evaluate the prognostic impact of abdominal fat area. Moreover, subcutaneous fat has been demonstrated to have cardiovascular benefits due to secretion of adiponectin, which has anti-inflammatory, insulin-sensitizing, and anti-atherogenic effects. ${ }^{30}$ These cardiovascular benefits could be another mechanism contributing to the aforementioned findings.

\section{Study Limitations}

This study had several limitations worth noting. A major limitation of the study was that it was a retrospective, single-center study with a small sample size. Furthermore, the clinical events were not adjudicated by an independent clinical event committee. Therefore, the results should be interpreted cautiously until verified in further studies. To our knowledge, however, this study is the first to report on the prognostic value of CT-derived SFA. As noted, SFA can be easily measured in patients undergoing TAVI, and is a more specific parameter of obesity than BMI, which has been previously established as a useful prognostic indicator in patients undergoing TAVI. Therefore, despite the limitations of the study, the present findings provide a novel prognostic indicator in patients undergoing TAVI, and may provide new insights into the concept of the obesity paradox. Further studies are needed to validate the current results and also to determine whether these findings are reproducible in other populations.

\section{Conclusions}

In patients undergoing TAVI, CT-derived SFA was an independent predictor of long-term adverse clinical outcome, whereas VFA was not. This simple and practical marker might be useful for predicting long-term clinical outcome in patients undergoing TAVI.

\section{Disclosures}

K.T. receives honoraria from Canon Medical Systems, Edwards Lifesciences, and Medtronic. M.Y. receives honoraria from Edwards Lifesciences. The other authors declare no conflicts of interest.

\section{References}

1. Holmes DR Jr, Brennan J, Rumsfeld JS, Dai D, O’Brien SM, Vemulapalli $S$, et al. Clinical outcomes at 1 year following transcatheter aortic valve replacement. JAMA 2015; 313: 10191028.

2. van der Boon RM, Chieffo A, Dumonteil N, Tchetche D, Van Mieghem NM, Buchanan GL, et al. Effect of body mass index on short- and long-term outcomes after transcatheter aortic valve implantation. Am J Cardiol 2013; 111: 231-236.

3. Yamamoto M, Mouillet G, Oguri A, Gilard M, Laskar M, Eltchaninoff $\mathrm{H}$, et al. Effect of body mass index on 30- and 365-day complication and survival rates of transcatheter aortic valve implantation (from the FRench Aortic National CoreValve and Edwards 2 [FRANCE 2] registry). Am J Cardiol 2013; 112: $1932-1937$

4. Konigstein M, Havakuk O, Arbel Y, Finkelstein A, Ben-Assa E, Leshem Rubinow E, et al. The obesity paradox in patients undergoing transcatheter aortic valve implantation. Clin Cardiol 2015; 38: 76-81.

5. Gonzalez-Ferreiro R, Munoz-Garcia AJ, Lopez-Otero D, Avanzas P, Pascual I, Alonso-Briales JH, et al. Prognostic value of body mass index in transcatheter aortic valve implantation: A "J"-shaped curve. Int J Cardiol 2017; 232: 342-347.

6. Lv W, Li S, Liao Y, Zhao Z, Che G, Chen M, et al. The 'obesity paradox' does exist in patients undergoing transcatheter aortic valve implantation for aortic stenosis: A systematic review and meta-analysis. Interact Cardiovasc Thorac Surg 2017; 25: 633642.

7. Sannino A, Schiattarella GG, Toscano E, Gargiulo G, Giugliano $\mathrm{G}$, Galderisi M, et al. Meta-analysis of effect of body mass index on outcomes after transcatheter aortic valve implantation. Am J Cardiol 2017; 119: 308-316.

8. Abramowitz Y, Chakravarty T, Jilaihawi H, Cox J, Sharma RP, Mangat G, et al. Impact of body mass index on the outcomes following transcatheter aortic valve implantation. Catheter Cardiovasc Interv 2016; 88: 127-134.

9. Gruberg L, Weissman NJ, Waksman R, Fuchs S, Deible R, Pinnow EE, et al. The impact of obesity on the short-term and long-term outcomes after percutaneous coronary intervention: The obesity paradox? J Am Coll Cardiol 2002; 39: 578-584.

10. Galal W, van Domburg RT, Feringa HH, Schouten O, Elhendy A, Bax JJ, et al. Relation of body mass index to outcome in patients with known or suspected coronary artery disease. Am J Cardiol 2007; 99: 1485-1490.

11. Roberts WC, Roberts CC, Vowels TJ, Ko JM, Filardo G, 
Hamman BL, et al. Effect of body mass index on survival in patients having aortic valve replacement for aortic stenosis with or without concomitant coronary artery bypass grafting. Am J Cardiol 2011; 108: 1767-1771.

12. Costanzo P, Cleland JG, Pellicori P, Clark AL, Hepburn D, Kilpatrick ES, et al. The obesity paradox in type 2 diabetes mellitus: Relationship of body mass index to prognosis: A cohort study. Ann Intern Med 2015; 162: 610-618.

13. Kim H, Kim J, Seo C, Lee M, Cha MU, Jung SY, et al. Body mass index is inversely associated with mortality in patients with acute kidney injury undergoing continuous renal replacement therapy. Kidney Res Clin Pract 2017; 36: 39-47.

14. Barba R, Marco J, Ruiz J, Canora J, Hinojosa J, Plaza S, et al. The obesity paradox in stroke: Impact on mortality and shortterm readmission. J Stroke Cerebrovasc Dis 2015; 24: 766-770.

15. Shah R, Gayat E, Januzzi JL Jr, Sato N, Cohen-Solal A, diSomma $\mathrm{S}$, et al. Body mass index and mortality in acutely decompensated heart failure across the world: A global obesity paradox. J Am Coll Cardiol 2014; 63: 778-785.

16. Tops LF, Wood DA, Delgado V, Schuijf JD, Mayo JR, Pasupati $\mathrm{S}$, et al. Noninvasive evaluation of the aortic root with multislice computed tomography implications for transcatheter aortic valve replacement. JACC Cardiovasc Imaging 2008; 1: 321 -330.

17. Gurvitch R, Wood DA, Leipsic J, Tay E, Johnson M, Ye J, et al. Multislice computed tomography for prediction of optimal angiographic deployment projections during transcatheter aortic valve implantation. JACC Cardiovasc Interv 2010; 3: 1157-1165.

18. Hayashida K, Bouvier E, Lefevre T, Hovasse T, Morice MC, Chevalier B, et al. Impact of CT-guided valve sizing on postprocedural aortic regurgitation in transcatheter aortic valve implantation. EuroIntervention 2012; 8: 546-555.

19. Binder RK, Webb JG, Willson AB, Urena M, Hansson NC, Norgaard BL, et al. The impact of integration of a multidetector computed tomography annulus area sizing algorithm on outcomes of transcatheter aortic valve replacement: A prospective, multicenter, controlled trial. J Am Coll Cardiol 2013; 62: 431 - 438.

20. Tokunaga K, Matsuzawa Y, Ishikawa K, Tarui S. A novel technique for the determination of body fat by computed tomog- raphy. Int J Obes 1983; 7: 437-445.

21. Seidell JC, Bakker CJ, van der Kooy K. Imaging techniques for measuring adipose-tissue distribution: A comparison between computed tomography and 1.5-T magnetic resonance. Am J Clin Nutr 1990; 51: 953-957.

22. Yoshizumi T, Nakamura T, Yamane M, Islam AH, Menju M, Yamasaki K, et al. Abdominal fat: Standardized technique for measurement at CT. Radiology 1999; 211: 283-286.

23. Garg L, Agrawal S, Pew T, Hanzel GS, Abbas AE, Gallagher MJ, et al. Psoas muscle area as a predictor of outcomes in transcatheter aortic valve implantation. Am J Cardiol 2017; 119: 457-460.

24. Rosenquist KJ, Pedley A, Massaro JM, Therkelsen KE, Murabito JM, Hoffmann U, et al. Visceral and subcutaneous fat quality and cardiometabolic risk. JACC Cardiovasc Imaging 2013; 6: 762-771.

25. Sato F, Maeda N, Yamada T, Namazui H, Fukuda S, Natsukawa T, et al. Association of epicardial, visceral, and subcutaneous fat with cardiometabolic diseases. Circ J 2018; 82: $502-508$.

26. Knudtson MD, Klein BE, Klein R, Shankar A. Associations with weight loss and subsequent mortality risk. Ann Epidemiol 2005; 15: 483-491.

27. Kunimura A, Ishii H, Uetani T, Aoki T, Harada K, Hirayama $\mathrm{K}$, et al. Impact of nutritional assessment and body mass index on cardiovascular outcomes in patients with stable coronary artery disease. Int $J$ Cardiol 2017; 230: 653-658.

28. Iwakami N, Nagai T, Furukawa TA, Sugano Y, Honda S, Okada A, et al. Prognostic value of malnutrition assessed by Controlling Nutritional Status score for long-term mortality in patients with acute heart failure. Int J Cardiol 2017; 230: 529536.

29. Yokoyama M, Watanabe T, Otaki Y, Watanabe K, Toshima T, Sugai $\mathrm{T}$, et al. Impact of objective malnutrition status on the clinical outcomes in patients with peripheral artery disease following endovascular therapy. Circ J 2018; 82: 847-856.

30. McCarty MF. A paradox resolved: The postprandial model of insulin resistance explains why gynoid adiposity appears to be protective. Med Hypotheses 2003; 61: 173-176. 